\title{
Effect of Deep Brain Stimulation on Female Parkinsonian Patients with Lower Urinary Tract Symptoms
}

\author{
Fuchao Liang ${ }^{1,2, *}$ \\ Yueqing Tang ${ }^{1,3}$, \\ Kaipeng $\mathrm{Bi}^{1,2}$ \\ Xuchen Liu ${ }^{2}$ \\ Chao $\mathrm{Li}^{4,5}$ \\ Si Chen ${ }^{6}$ \\ Chao Zhang (iD) ${ }^{4,5}$ \\ Lei Yan' \\ Zhonghua $\mathrm{Xu}{ }^{\prime}$ \\ Ning Yang (iD ${ }^{4,5,7}$
}

'Department of Urology, Qilu Hospital of Shandong University, Jinan, People's Republic of China; ${ }^{2}$ School of Medicine, Cheeloo College of Medicine, Shandong University, Jinan, People's Republic of China; ${ }^{3}$ Department of Andrology, Shandong University, Jinan, People's Republic of China; ${ }^{4}$ Department of Neurosurgery, Qilu Hospital of Shandong University and Institute of Brain and BrainInspired Science, Shandong University, Jinan, People's Republic of China; ${ }^{5}$ Shandong Key Laboratory of Brain Function Remodeling, Jinan, 2500 I2, People's Republic of China; ${ }^{6}$ Department of Neurology, Qilu Hospital of Shandong University, Jinan, People's Republic of China; ${ }^{7}$ Department of Epidemiology and Health Statistics, School of Public Health, Shandong University, Jinan, 2500I2, People's Republic of China

*These authors contributed equally to this work
Purpose: This study aimed to investigate the characteristics of urodynamics in female parkinsonian patients with lower urinary tract symptoms (LUTS) and evaluate the outcomes of deep brain stimulation (DBS) based on overactive bladder (OAB) scores and quality of life (QOL) scores.

Patients and Methods: Urodynamic assessment was performed in 20 female parkinsonian patients with LUTS. OAB scores and QOL scores were collected before and 6 months after DBS treatment (The target of DBS is STN). We evaluated the related factors affecting QOL score and also the changes in QOL score after DBS treatment.

Results: The mean age of 20 patients was $60.6 \pm 6.3$ years, with $65 \%$ of patients identified with $\mathrm{OAB}$ in urodynamic studies. The mean OAB total score and QOL score at baseline were $7.3 \pm 3.7$ and $5.0 \pm 1.1$, respectively. Four items in the $\mathrm{OAB}$ score were found to be independent factors and they influenced the baseline QOL scores. Six months after DBS treatment, the OAB total score and the QOL score were significantly decreased $(3.7 \pm 3.2$ ( $p=$ $0.002)$ and $3.4 \pm 1.4(p<0.001)$, respectively). Improvements in OAB item 2 (nocturia) and item 3 (urgency) scores were found to be independent factors, which had an influence on the improvement in QOL scores from multivariate analysis. Improvement of OAB item 2 (nocturia) exhibited the greatest influence on improvement in the QOL score. After DBS treatment, $40 \%$ of the patients showed significant improvement in urination symptoms $(\geq 2$ points reduction in QOL score), 30\% of the patients showed mild improvement (1 point reduction in QOL score), and 30\% patients showed no improvement (no change in QOL score). No patients exhibited worsening urination symptoms.

Conclusion: Most parkinsonian female patients with lower urinary tract symptoms were diagnosed with OAB. DBS treatment improved OAB symptoms. Improvement of nocturia and urgency positively impacts the QOL of female parkinsonian patients.

Keywords: Parkinson's disease, urodynamics, OAB score, QOL score
Correspondence: Ning Yang

Department of Neurosurgery, Qilu Hospital of Shandong University and Institute of Brain and Brain-Inspired Science, Shandong University, Jinan, People's Republic of China

$\mathrm{Tel} / \mathrm{Fax}+86$ 53I-82I6663I

Email yangning@sdu.edu.cn

Zhonghua $\mathrm{Xu}$

Department of Urology, Qilu Hospital of

Shandong University, Jinan, People's Republic of

China

Tel/Fax +86 531-8216670I

Email xuzhonghua1963@163.com

\section{Introduction}

Parkinson's disease (PD) is a common neurodegenerative disease, and motor symptoms are characterized by resting tremor, rigidity, postural instability and bradykinesia. Lower urinary tract symptoms (LUTS) are one of the most common autonomic symptoms in PD, which include urinary urgency, frequency, and nocturia, occurring with or without urgency incontinence. ${ }^{1-3}$ Despite the impact of LUTS on a patient's quality of life, little is known about its pathogenesis and posttreatment response. ${ }^{4}$ Neurologists most often focus on motor symptoms and ignore 
the urinary symptoms of these patients. In fact, improvement in LUTS can greatly enhance the quality of life (QOL) of patients.

Urodynamic study is a powerful tool for evaluating urinary dysfunction in PD patients. ${ }^{5}$ QOL scores are widely used for evaluation of quality of life caused by LUTS and it represents patients' satisfaction with their current quality of life. ${ }^{6-9}$ Overactive bladder (OAB) scores consist of four items (OAB-1: frequency; OAB-2: nocturia; OAB-3: urgency; OAB-4: urgency incontinence) and are used to evaluate the status of patients with overactive bladder by questionnaire scoring. $\mathrm{OAB}$ scores can assist in the clinical prediction of patients with overactive bladder. ${ }^{10,11}$

Deep brain stimulation (DBS) has been used as a surgical treatment for motor symptoms in PD, but its effectiveness in improving urination dysfunction is still controversial. ${ }^{12-14}$ This study evaluated urination dysfunction in female parkinsonian patients with lower urinary tract symptoms based on urodynamic examination. The therapeutic effect of DBS was evaluated by analyzing the changes in OAB scores and QOL scores before and after DBS. Our results indicate that DBS may improve LUTS in female parkinsonian patients and thus, may lead to a better understanding of voiding dysfunction in these patients.

\section{Materials and Methods}

\section{Ethics Statement and Informed Consent}

This study was conducted in accordance with the Declaration of Helsinki, and all patients provided written informed consent, and the study was approved by the Ethical Review Board of Qilu Hospital (Ethics approval number is KYLL-2020-463), Shandong University.

\section{Data Sources}

We reviewed female parkinsonian patients with LUTS referred to the urology department at the Qilu Hospital of Shandong University for urodynamic studies during the period between June 2017 and February 2021. Exclusion criteria were the following: 1) urethral stricture, uterine prolapse, bladder stones, or recurrent urinary tract infections; 2) cerebrovascular disease or neurological disorders other than Parkinson's disease; 3) pregnancy and previous gynecological surgeries; and 4) Parkinson's disease patients without subsequent DBS treatment. Thirty-six female parkinsonian patients with LUTS were screened from the data (based on our exclusion criteria), and then we excluded 16 patients who were not treated with DBS, finally, 20 patients meeting the criteria were included, and all of them satisfied the clinical diagnostic criteria of the British Parkinson's Disease Society brain Bank. OAB scores and QOL scores were collected before and 6 months after DBS treatment.

\section{Urodynamic Study and Deep Brain Stimulation}

A Laborie urodynamic examination instrument was used for the examination, which was performed in accordance with the urodynamic examination criteria established by the International Continence Society (ICS). ${ }^{15}$ The patients were maintained in a semi-sitting position, with the F8 dualchamber urethropiezometric pipe placed through the urethra to assess the bladder pressure and the single-chamber piezometric pipe placed through the anus to assess the abdominal pressure. All examinations were performed according to ICS urodynamic specifications. The sensor was calibrated in vitro to zero according to the ICS calibration standard. Subsequently, the distal end of the perfusion and pressure tube system was connected to a three-chamber pressure tube. To check the conductivity and tightness of the piezometric piping system, the patients were asked to cough. After zeroing, room temperature $\left(20^{\circ} \mathrm{C}\right)$ saline was perfused into the bladder at a speed of $10-40 \mathrm{~mL} / \mathrm{min}$, and the bladder stability, sensation, compliance, and capacity during the perfusion were recorded. When the patients reached the urination limit, they were asked to urinate at the $45^{\circ}$ inclined seating position. The detrusor pressure, bladder pressure, and flow rate were measured simultaneously, while the urine flowed into a urine collection device. The parameters of maximum urinary flow rate $(\mathrm{Qmax})(\mathrm{mL} / \mathrm{s})$, detrusor pressure at maximum flow rate (PdetQmax) $\left(\mathrm{cmH}_{2} \mathrm{O}\right)$, postvoid residual (PVR) $(\mathrm{mL})$, and bladder compliance were recorded. Impaired anal muscle strength was assessed on the basis of anal reflexes and autonomic anal contraction strength. Detrusor dysfunction, bladder compliance and overactive bladder were defined based on the standardized report for the terminology of lower urinary tract function by the ICS. ${ }^{16}$ The target of DBS is STN, and its parameter adjustments are as follows: amplitude is $0-3.6 \mathrm{~V}$, pulse width is $60 / 90 / 120 u s$, frequency is $130-185 \mathrm{~Hz}$, and the stimulation pattern is monopolar stimulation.

\section{Statistical Analysis}

All statistical analyses were performed using IBM SPSS Statistics for Windows, Version 25.0 (IBM Corp.; Armonk, 
NY, USA) and R software version 3.6.1. Specific analysis parameters were age, Qmax, PdetQmax, PVR, bladder compliance, anal muscle strength, QOL score and $\mathrm{OAB}$ score. Categorical data and numeric parameters were compared using the Chi-square test and the independent $t$-test, respectively. Data are presented as the mean \pm standard deviation. $\mathrm{R}$ software was performed to analyze baseline characteristics of various indicators of patients before and after DBS. LM package in $\mathrm{R}$ software was used to construct unit and multivariate regression models to study the relevant influencing factors of the QOL score and changes in the QOL score after DBS treatment. Spearman correlation analysis was performed to analyze the correlation between $\mathrm{OAB}$ scores in urodynamic examination and $\mathrm{OAB}$ scores, and verified the predictability of $\mathrm{OAB}$ scores. $P<0.05$ was considered statistically significant, and all hypotheses were evaluated in a two-sided manner.

\section{Results}

A total of 36 female parkinsonian patients with LUTS were evaluated by urodynamic study. A total of 20 patients were included in final analysis after exclusion of 16 patients who had not received DBS treatment. All patients had urine storage phase symptoms (one or more symptoms of frequency, nocturia, urgency and urgency incontinence), and five patients had urination symptoms (weakness of urine) at the same time. Baseline characteristics of the study population are presented in Table 1 . The mean age of the 20 patients was $60.6 \pm 6.3$ years, the mean Qmax, $13.3 \mathrm{~mL} / \mathrm{s}$, the mean PdetQmax, $29.1 \mathrm{cmH}_{2} \mathrm{O}$, and the mean PVR, $29.8 \mathrm{~mL}$. Fifteen (75\%) patients had decreased anal muscle strength, $1(5 \%)$ patient had decreased bladder compliance, and $13(65 \%)$ patients were found with $\mathrm{OAB}$ based on urodynamic study. The mean OAB total scores and QOL scores at baseline were 7.3 \pm 3.7 and 5.0 \pm 1.1 , respectively. After 6 months of DBS treatment, OAB total scores and QOL scores were significantly decreased $(3.7 \pm 3.2(p=0.002)$ and $3.4 \pm 1.4 \quad(p<0.001)$, respectively).

\section{Factors Influencing Baseline QOL Scores}

Univariate regression analysis showed that $\mathrm{OAB}$, anal muscle strength and four items of the $\mathrm{OAB}$ score were correlated to baseline QOL scores (Table 2). However, age, Qmax, bladder compliance, PVR and PdetQmax were not significant factors affecting QOL scores. Multivariate regression analysis showed that $\mathrm{OAB}$ item 1 (frequency), $\mathrm{OAB}$ item 2 (nocturia), $\mathrm{OAB}$ item 3 (urgency), and $\mathrm{OAB}$ item 4 (urgency incontinence) were significant factors influencing QOL scores.

\section{Factors Influencing QOL Scores}

Univariate and multivariate analysis results for factors that influenced changes in QOL scores are presented in Table 3. Changes in $\mathrm{OAB}$ score item 2 (nocturia) and item 3 (urgency) were independent factors that influenced change in QOL scores after DBS treatment. Changes in $\mathrm{OAB}$ item 2 (nocturia) had the greatest influence on the improvement in QOL scores. As the score of OAB item 2 (nocturia) decreased by 1 point, the QOL score decreased by 0.915 point.

Table I Baseline Characteristics and Changes After DBS for 6 Months

\begin{tabular}{|l|c|c|c|}
\hline Variables & Baseline & 6 Months Follow-Up & P value \\
\hline Age (years) & $60.6 \pm 6.3$ & & \\
Qmax (mL/s) & $13.3 \pm 7.2$ & & \\
Bladder compliance (normal) & $19(95 \%)$ & \\
OAB (exist) & $13(65 \%)$ & \\
PdetQmax (mL/s) & $29.1 \pm 10.7$ & & \\
Anal muscle strength (weak) & $15(75 \%)$ & & \\
PVR (mL) & $29.8 \pm 47.0$ & & \\
OAB score item 1 & $1.2 \pm 0.7$ & $0.5 \pm 0.6$ & 0.002 \\
OAB score item 2 & $2.3 \pm 1.1$ & $1.3 \pm 1.1$ & 0.006 \\
OAB score item 3 & $2.4 \pm 1.6$ & $1.3 \pm 1.5$ & 0.042 \\
OAB score item 4 & $1.6 \pm 1.6$ & $0.7 \pm 1.3$ & 0.081 \\
Total OAB score & $7.3 \pm 3.7$ & $3.7 \pm 3.2$ & 0.002 \\
QOL score & $5.0 \pm 1.1$ & $3.4 \pm 1.4$ & $<0.001$ \\
\hline
\end{tabular}

Abbreviations: Qmax, maximum urinary flow rate; OAB, overactive bladder; PdetQmax, detrusor pressure at maximum flow rate; PVR, post-void residual; QOL, quality of life. 
Table 2 Factors Influencing QOL Scores

\begin{tabular}{|l|r|r|c|c|}
\hline \multirow{2}{*}{ Variables } & \multicolumn{2}{|c|}{ Univariate } & \multicolumn{2}{c|}{ Multivariate } \\
\cline { 2 - 5 } & \multicolumn{1}{|c|}{ B } & $P$ & $B$ & $P$ \\
\hline Age & 0.015 & 0.730 & & \\
Qmax & -0.053 & 0.144 & & \\
Bladder compliance & $<0.001$ & 1.000 & & \\
OAB & 1.10 & 0.033 & -0.114 & 0.655 \\
PdetQmax & 0.031 & 0.210 & & \\
Anal muscle strength & -1.600 & 0.003 & -0.356 & 0.206 \\
PVR & 0.008 & 0.138 & & \\
OAB score item I & 1.196 & $<0.001$ & 0.499 & 0.027 \\
OAB score item 2 & 0.782 & $<0.001$ & 0.285 & 0.021 \\
OAB score item 3 & 0.534 & $<0.001$ & 0.283 & 0.005 \\
OAB score item 4 & 0.353 & 0.020 & 0.150 & 0.028 \\
\hline
\end{tabular}

Abbreviations: $B$, regression coefficient; $P, P$ value; $Q \max$, maximum urinary flow rate; $\mathrm{OAB}$, overactive bladder; PdetQmax, detrusor pressure at maximum flow rate; PVR, post-void residual.

\section{Assessment of Changes in QOL Scores}

After 6 months of DBS treatment, according to the changes in the QOL scores and the feelings of symptoms improvement reported by the patients, we obtained that $40 \%$ of patients showed significant improvement in urination symptoms ( $\geq 2$ points reduction in QOL score), $30 \%$ of the patients showed mild improvement ( 1 point reduction in QOL score), and 30\% of patients showed no improvement (no change in QOL score). No patients exhibited worsening urination symptoms. Thirteen patients were diagnosed with OAB through urodynamic study. After DBS treatment, eight of these patients showed significant improvement in urination (QOL score decreased $\geq 2$ points), two patients showed slight improvement (QOL score decreased 1 point), and three patients showed no improvement (QOL score did not change). Seven patients were diagnosed without $\mathrm{OAB}$ through urodynamic study. Although none of these seven patients showed significant improvement (QOL score decreased by $\geq 2$ points) after DBS treatment, four patients showed slight improvement (QOL score decreased by 1 point) and three patients showed no improvement (No change in QOL score) (Table 4).

\section{A Positive Correlation Between OAB in Urodynamic Study and OAB Scores}

Spearman correlation analysis revealed a significant positive correlation between $\mathrm{OAB}$ results from the urodynamic study and OAB scores in QOL (correlation coefficient $R=0.478 ; P=0.03$ ). The presence of $\mathrm{OAB}$ in the urodynamic study predicted relatively high QOL scores. Changes in OAB scores tended to be associated with improvement in urination symptoms.

\section{Discussion}

Parkinson's disease is a common degenerative neurologic disease, and motor symptoms are the main manifestation. Non-motor symptoms, such as hyposmia, insomnia, depression and frequent urination, also seriously affect the QOL of PD patients. ${ }^{17}$ Although DBS is effective in improving PD symptoms, especially motor symptoms, such as tremor, rigidity and bradykinesia, there has been no in-depth study on the impact of the DBS on non-motor

Table 3 Factors Influencing Change in QOL Scores

\begin{tabular}{|c|c|c|c|c|}
\hline \multirow[b]{2}{*}{ Variables } & \multicolumn{2}{|l|}{ Univariate } & \multicolumn{2}{|l|}{ Multivariate } \\
\hline & B & $P$ & B & $P$ \\
\hline Age & -0.034 & 0.593 & & \\
\hline Qmax & -0.016 & 0.774 & & \\
\hline Bladder compliance & 0.684 & 0.700 & & \\
\hline $\mathrm{OAB}$ & 1.659 & 0.029 & -0.322 & 0.378 \\
\hline PdetQmax & 0.039 & 0.280 & & \\
\hline Anal muscle strength & -1.13 & 0.194 & & \\
\hline PVR & 0.009 & 0.292 & & \\
\hline Change in $O A B$ score item I & 1.069 & 0.036 & 0.389 & 0.104 \\
\hline Change in $O A B$ score item 2 & 1.555 & $<0.001$ & 0.915 & $<0.001$ \\
\hline Change in $O A B$ score item 3 & 0.966 & $<0.001$ & 0.450 & 0.011 \\
\hline Change in $O A B$ score item 4 & 0.655 & 0.013 & 0.116 & 0.338 \\
\hline
\end{tabular}

Abbreviations: $B$, regression coefficient; $P$, $P$ value; $Q m a x$, maximum urinary flow rate; $O A B$, overactive bladder; PdetQmax, detrusor pressure at maximum flow rate; PVR, post-void residual. 
Table 4 Assessment of Improvement Based on Changes in QOL Scores

\begin{tabular}{|l|c|c|l|}
\hline $\begin{array}{l}\text { Group According to the Change in } \\
\text { QOL Scores }\end{array}$ & $\begin{array}{c}\text { OAB Confirmed by } \\
\text { Urodynamic Study }\end{array}$ & $\begin{array}{c}\text { OAB Not Confirmed by } \\
\text { Urodynamic Study }\end{array}$ & Total \\
\hline$\geq 2$ Points reduction in QOL scores & $8(61.5 \%)$ & $0(0 \%)$ & $8(40 \%)$ \\
I Point reduction in QOL scores & $2(15.4 \%)$ & $4(57.1 \%)$ & $6(30 \%)$ \\
No change in QOL scores & $3(23.1 \%)$ & $3(42.9 \%)$ & $6(30 \%)$ \\
Total number of patients & $13(65 \%)$ & $7(35 \%)$ & $20(100 \%)$ \\
\hline
\end{tabular}

Abbreviations: OAB, overactive bladder; QOL, quality of life.

symptoms, especially for LUTS. LUTS includes storage symptoms (urinary frequency, nocturia, urinary urgency, and urgency incontinence) and voiding symptoms (slow and/or interrupted stream, terminal dribble, hesitancy and training). ${ }^{18}$ Previous urodynamic studies have shown that a high proportion of Parkinson's patients have OAB. ${ }^{19}$ $\mathrm{OAB}$ symptoms are characterized by urgent urination and can be accompanied by frequent urination, nocturia, and urgency incontinence, which can seriously interfere with and impair health-related quality of life. ${ }^{20}$

Female parkinsonian patients were selected as the study group to eliminate problems associated with the male prostate. Benign prostatic hyperplasia (BPH) leads to bladder outlet obstruction, and LUTS may be secondary to $\mathrm{BPH}^{21}$ When male PD patients have abnormal urodynamic parameters, we cannot attribute them to $\mathrm{PD}$ or $\mathrm{BPH}$ with certainty. Therefore, we selected females to study the effect of PD on patients' urinary storage and urination dysfunction, and thus eliminate confounding factors as much as possible. However, we also excluded urethral stricture and uterine prolapse from the inclusion criteria, which could also lead to bladder outlet obstruction in females.

In our study, all patients had symptoms of urination storage, and only five patients had urination symptoms at the same time. Urodynamic study showed that most patients had $\mathrm{OAB}$, and $\mathrm{OAB}$ often presented as symptoms of urine storage, which indicated that LUTS in female parkinsonian patients mainly occurred during the urine storage phase.

When these patients sought out treatment at the hospital for LUTS, their quality of life had been disrupted and treatments were required. Most patients showed OAB and anal sphincter damage on examination, which may be caused by neurogenic injury. The results of the patient's urodynamic examination are more likely to be an abnormal indicator of neuronal injury. Our study also showed that frequency, nocturia, urgency and urgency incontinence are important factors affecting PD patient quality of life, and these symptoms were mainly associated with the urine storage period. Previous studies of patients with LUTS also support our observations that symptoms of urination storage were more likely to affect patients QOL than voiding symptoms. ${ }^{22}$ Thus, the conclusions of our study are consistent with previous results.

The pathophysiology of OAB symptoms in PD may be disruption of the dopamine D1-GABAergic direct pathway and its GABAergic collateral to the micturition circuit, resulting in urination reflex and loss of inhibition of OAB symptoms. OAB symptoms correlate in severity with urodynamic abnormalities and dopaminergic deficit on dopamine transporter scans. ${ }^{18,23}$ Despite the high incidence of LUTS and its impact on QOL, current treatment options for PD patients are limited. Antimycin is the firstline treatment for OAB symptoms. Intradermal injection of botulinum toxin is also effective in OAB, but it is associated with the risk of urinary retention. Finally, desmopressin is effective in the treatment of nocturnal polyuria, which is common in PD patients. ${ }^{24}$ If there is no significant $\mathrm{OAB}$ on urodynamic study, pharmacological treatment may not significantly improve LUTS. DBS is therefore a promising minimally invasive surgical method for the treatment of PD. However, few studies have been performed to examine urination storage and urination dysfunction in Parkinson's patients, and thus, the conclusions are still controversial. ${ }^{12,14}$

DBS can improve degenerated neural electrical activity and restore neurotransmitters and motor control circuits to a relatively normal functional state. We speculated that DBS can improve signal transduction of neural pathways, thus relieving $\mathrm{OAB}$ and improving LUTS. Our study showed that after DBS treatment, the QOL of a considerable number of patients improved, and the change in nocturia and urgency symptoms was the most important factor influenced on the 
improvement in QOL. We found that the QOL of 77\% of the patients with $\mathrm{OAB}$, as assessed by preoperative urodynamic study, was improved after DBS treatment, with $62 \%$ of these patients showing significant improvement in QOL. QOL in $57 \%$ of the patients without $\mathrm{OAB}$, as assessed by preoperative urodynamic study, improved slightly after DBS treatment. Correlation analysis showed a strong correlation between $\mathrm{OAB}$, as determined in urodynamic study, and OAB scores. Therefore, we speculated that DBS treatment may improve $\mathrm{OAB}$ symptoms in patients diagnosed with $\mathrm{OAB}$ in the preoperative urodynamic study and ultimately the patient's QOL.

The study also has several limitations. First, the sample size is small. Only 20 female patients were included in the study due to the robust inclusion criteria. However, these 20 patients were all evaluated by urologists through urodynamic study and received DBS treatment. Thus, although our sample size was limited, we carefully managed the cases in order to achieve reliable and instructive results. Finally, these patients were not evaluated through urodynamic study after DBS, which may help us to further understand the nature of their development and treatment. On the other hand, we hope that this conclusion can provide a few guidance for the treatment of female parkinsonian patients with lower urinary tract symptoms in the future.

\section{Conclusion}

Lower urinary tract symptoms of female parkinsonian patients are mainly observed in the urine storage phase as a majority of these patients have OAB. DBS treatment can improve OAB symptoms. Improvement of nocturia and urgency is important for the QOL of patients, and improvement in nocturia had the greatest influence on the improvement in QOL scores.

\section{Funding}

This work was supported by the Natural Science Foundation of China Grant (81702475) and the Jinan Science and Technology Bureau of Shandong Province (201704083).

\section{Disclosure}

The authors report no conflicts of interest in this work.

\section{References}

1. Sakakibara R, Panicker J, Finazzi-Agro E, et al. A guideline for the management of bladder dysfunction in Parkinson's disease and other gait disorders. Neurourol Urodyn. 2016;35:551-563. doi:10.1002/ nau. 22764
2. Sakakibara R, Tateno F, Yamamoto T, et al. Urological dysfunction in synucleinopathies: epidemiology, pathophysiology and management. Clin Auton Res. 2018;28(1):83-101. doi:10.1007/ s10286-017-0480-0

3. Kim H-S, Cheon S-M, Seo J-W, et al. Nonmotor symptoms more closely related to Parkinson's disease: comparison with normal elderly. J Neurol Sci. 2013;324:70-73. doi:10.1016/j.jns.2012.10.004

4. McDonald C, Winge K, Burn DJ. Lower urinary tract symptoms in Parkinson's disease: prevalence, aetiology and management. Parkinsonism Relat Disord. 2017;35:8-16. doi:10.1016/j. parkreldis.2016.10.024

5. Shin JH, Park KW, Heo KO, et al. Urodynamic study for distinguishing multiple system atrophy from Parkinson disease. Neurology. 2019;93:e946-e53. doi:10.1212/WNL.0000000000008053

6. Sarma AV, Wei JT. Clinical practice. Benign prostatic hyperplasia and lower urinary tract symptoms. $N$ Engl J Med. 2012;367:248-257. doi:10.1056/NEJMcp1106637

7. Barry MJ, Fowler FJ, O'Leary MP, et al. The American Urological Association symptom index for benign prostatic hyperplasia. The Measurement Committee of the American Urological Association. J Urol. 1992;148:1549-1557. doi:10.1016/S0022-5347(17)36966-5

8. Barry MJ. Evaluation of symptoms and quality of life in men with benign prostatic hyperplasia. Urology. 2001;58(6):25-32. doi:10.1016/S0090-4295(01)01300-0

9. Yoshimura K, Arai Y, Ichioka K, et al. Symptom-specific quality of life in patients with benign prostatic hyperplasia. Int $J$ Urol. 2002;9:485-490. doi:10.1046/j.1442-2042.2002.00503.x

10. Arribillaga LC, Ledesma M, Montedoro A, et al. OAB score: a clinical model that predicts the probability of presenting overactive detrusor in the urodynamic study. Int Braz J Urol. 2018;44:348-354. doi:10.1590/s1677-5538.ibju.2017.0213

11. Matza LS, Thompson CL, Krasnow J, et al. Test-retest reliability of four questionnaires for patients with overactive bladder: the overactive bladder questionnaire (OAB-q), patient perception of bladder condition (PPBC), urgency questionnaire (UQ), and the primary $\mathrm{OAB}$ symptom questionnaire (POSQ). Neurourol Urodyn. 2005;24:215-225. doi:10.1002/nau.20110

12. Kalia LV, Lang AE. Parkinson's disease. Lancet (London, England). 2015;386:896-912. doi:10.1016/S0140-6736(14)61393-3

13. Finazzi-Agrò E, Peppe A, D'Amico A, et al. Effects of subthalamic nucleus stimulation on urodynamic findings in patients with Parkinson's disease. J Urol. 2003;169:1388-1391. doi:10.1097/01. ju.0000055520.88377.dc

14. Witte LP, Odekerken VJJ, Boel JA, et al. Does deep brain stimulation improve lower urinary tract symptoms in Parkinson's disease? Neurourol Urodyn. 2018;37:354-359. doi:10.1002/nau.23301

15. Schäfer W, Abrams P, Liao L, et al. Good urodynamic practices: uroflowmetry, filling cystometry, and pressure-flow studies. Neurourol Urodyn. 2002;21(3):261-274. doi:10.1002/nau.10066

16. Abrams P, Cardozo L, Fall M, et al. The standardisation of terminology in lower urinary tract function: report from the standardisation sub-committee of the International Continence Society. Urology. 2003;61:37-49. doi:10.1016/S0090-4295(02)02243-4

17. Pont-Sunyer C, Hotter A, Gaig C, et al. The onset of nonmotor symptoms in Parkinson's disease (the ONSET PD study). Mov Disord. 2015;30:229-237. doi:10.1002/mds.26077

18. Sakakibara R, Tateno F, Kishi M, et al. Pathophysiology of bladder dysfunction in Parkinson's disease. Neurobiol Dis. 2012;46:565-571. doi:10.1016/j.nbd.2011.10.002

19. Xue P, Wang T, Zong H, et al. Urodynamic analysis and treatment of male Parkinson's disease patients with voiding dysfunction. Chin Med J. 2014;127:878-881.

20. Coyne KS, Payne C, Bhattacharyya SK, et al. The impact of urinary urgency and frequency on health-related quality of life in overactive bladder: results from a national community survey. Value Health. 2004;7:455-463. doi:10.1111/j.1524-4733.2004.74008.x 
21. Biardeau X, Elkoushy MA, Aharony S, et al. Is multichannel urodynamic assessment necessary before considering a surgical treatment of BPH? Pros and cons. World J Urol. 2016;34:463-469. doi:10.1007/s00345-015-1647-8

22. McVary KT, Peterson A, Donatucci CF, et al. Use of structural equation modeling to demonstrate the differential impact of storage and voiding lower urinary tract symptoms on symptom bother and quality of life during treatment for lower urinary tract symptoms associated with benign prostatic hyperplasia. $J$ Urol. 2016;196:824-830. doi:10.1016/j.juro.2016.04.062
23. Winge K, Friberg L, Werdelin L, et al. Relationship between nigrostriatal dopaminergic degeneration, urinary symptoms, and bladder control in Parkinson's disease. Eur J Neurol. 2005;12:842-850. doi:10.1111/j.1468-1331.2005.01087.x

24. Batla A, Tayim N, Pakzad M, et al. Treatment options for urogenital dysfunction in Parkinson's Disease. Curr Treat Options Neurol. 2016;18:45. doi:10.1007/s11940-016-0427-0

\section{Publish your work in this journal}

Neuropsychiatric Disease and Treatment is an international, peerreviewed journal of clinical therapeutics and pharmacology focusing on concise rapid reporting of clinical or pre-clinical studies on a range of neuropsychiatric and neurological disorders. This journal is indexed on PubMed Central, the 'PsycINFO' database and CAS, and is the official journal of The International Neuropsychiatric Association (INA). The manuscript management system is completely online and includes a very quick and fair peer-review system, which is all easy to use. Visit http://www.dovepress.com/testimonials.php to read real quotes from published authors. 